\title{
El cuatrimestre de la COVID-19 en la asignatura de Arquitectura de Computadores: reflexiones sobre la incidencia de la pandemia en la docencia universitaria
}

\author{
Julio Ortega, Mancia Anguita \\ Departamento de Arquitectura y Tecnología de Computadores \\ E.T.S.I.I.T., Universidad de Granada \\ \{jortega, manguita\}@ugr.es
}

\begin{abstract}
Resumen. La pandemia de COVID-19 ha afectado de forma significativa a la docencia de las asignaturas universitarias del segundo cuatrimestre del curso 2019-20 y del primer cuatrimestre del 2020-2021. Así, se ha requerido usar plataformas para impartir las asignaturas a través de videoconferencias, grabar las clases, y elaborar y realizar exámenes no presenciales. En particular, las circunstancias que acompañan la evaluación no presencial han generado situaciones que deben analizarse en el ámbito de la relación estudiante-profesor y estudiante-estudiante, precisamente en un contexto en el que la limitada interacción a distancia entre docentes y estudiantes ha dificultado en muchos casos alcanzar el nivel de confianza necesario para lograr los niveles de aprendizaje que se conseguían a través de clases presenciales. Esta situación se ha sido evidente en asignaturas con grupos muy numerosos, tal y como ocurre en el caso de la asignatura Arquitectura de Computadores del grado en Ingeniería Informática de la Universidad de Granada, que se considera en este artículo.
\end{abstract}

Palabras clave: Arquitectura de Computadores, Docencia no presencial Evaluación del aprendizaje.

\begin{abstract}
The COVID-19 pandemic has significantly affected the teaching of university subjects in the second quarter of the 2019-20 academic year and the first quarter of 2020-2021. Thus, it has been necessary to use technological platforms to teach the subjects through videoconferences, to record the classes, and to prepare and carry out remote exams. In particular, the circumstances that accompany the remote assessment have generated situations that must be analysed in the area of the student-teacher and student-student relationships, precisely in a context in which the limited distance interaction between the teacher and his or her students has made it difficult in many cases to achieve the level of confidence achieved through face-to-face classes. This situation has been clearly evident in subjects with very large groups, as has been the case with the Computer Architecture subject of the degree in Computer Engineering at the University of Granada, which is considered in this paper.
\end{abstract}

Keywords: Computer architecture, face-to-face classes, remote assessment, remote teaching. 


\section{Introducción}

En artículos previos publicados en esta revista [1-4] se han considerado distintos tópicos relacionados con la docencia de la arquitectura de computadores como asignatura de grado en ingeniería informática Así, en [1] se reflexiona acerca de los contenidos de una asignatura de arquitectura de computadores de 6 créditos ECTS (European Credit Transfer System), créditos del EEES (Espacio Europeo de Educación Superior) o créditos de la reforma Bolonia, y en [2] se evalúan algunos resultados y conclusiones que se podían derivar tras varios años de docencia de la asignatura. Finalmente, en [4] se actualiza y completa la revisión de contenidos de Arquitectura de Computadores describiendo su situación al comenzar la década de 2020, analizando los nuevos requisitos de las interfaces que definen la jerarquía de capas propia de los sistemas de cómputo (fundamentalmente en cuanto a seguridad, consumo energético, y aprovechamiento del paralelismo heterogéneo). Si bien el propósito de [4] era poner de manifiesto los cambios que deberían introducirse en la enseñanza superior de la Ingeniería de Computadores, el artículo se centraba fundamentalmente en la justificación de los contenidos que definen la materia de las asignaturas de ingeniería de computadores, sin apenas considerar las cuestiones relacionadas con la metodología docente. Precisamente, en otros trabajos previos [2] se reflexionaba sobre las consecuencias que las tecnologías informáticas pueden tener sobre el aprendizaje a partir de algunos resultados de la neurociencia, sobre la motivación en el estudio de las asignaturas de ingeniería de computadores [3], y sobre los beneficios que puede reportar al aprendizaje incluir elementos de la historia de los computadores para construir un relato de la asignatura que ayude a contextualizar los conceptos que se van abordando dentro del desarrollo de la disciplina [3]. En la línea de lo que se hacía en [4] en relación con los contenidos de la materia, en el presente trabajo se vuelven a considerar cuestiones metodológicas, y de motivación al comienzo de la década de 2020, pero ahora teniendo en cuenta las circunstancias derivadas de la pandemia de la COVID-19, que nos afecta desde el segundo cuatrimestre del curso 2019-2020. Precisamente, la adaptación de la docencia presencial a un contexto no presencial en un lapso de tiempo reducido, ha introducido una serie de problemas a tener en cuenta, así como la necesidad de reflexionar acerca de ciertas cuestiones relacionadas con la necesidad de promover ciertos valores éticos en la formación de nuestros estudiantes.

En efecto, recordaremos el segundo cuatrimestre del curso 2019/2020 por la incidencia de la pandemia en muchos aspectos de nuestra vida, y la docencia universitaria no ha sido una excepción entre las actividades que se han visto afectadas significativamente por esta pandemia, que ocasionó la declaración del estado de alarma en nuestro país, con el consiguiente confinamiento de la población y la suspensión de la actividad docente presencial desde mediados de marzo de 2020. Prácticamente todo el segundo cuatrimestre del curso 2019/2020 se impartió de forma no presencial (término que es el que se ha extendido, aunque quizá sea más adecuado usar el más tradicional “a distancia”). Impartir asignaturas de un plan de estudios universitario de forma no presencial o a distancia, implica satisfacer ciertos requisitos en cuanto a la explicación de los contenidos, el tipo de material que se proporciona a los estudiantes, y la evaluación de los conceptos asimilados y las competencias 
alcanzadas. Esta adaptación de la docencia se tuvo que hacer en su momento con cierta premura y no siempre se han adoptado herramientas y estrategias adecuadas, para la docencia a distancia.

Así, la Sección 2 de este artículo describe la metodología seguida para impartir la asignatura de Arquitectura de Computadores en el grado de Ingeniería Informática, según las directrices del EEES (reforma de Bolonia). En la Sección 3 se analizan las consecuencias del paso a la docencia no presencial tras el confinamiento decretado durante la primera oleada de la pandemia de la COVID-19. Una de las consecuencias más conflictivas y complicadas de implementar ha sido la evaluación no presencial de los aprendizajes, tal y como se muestra en la Sección 4, junto con su deriva ética. Finalmente, la Sección 5 proporciona las conclusiones del artículo junto con algunas propuestas para el futuro.

\section{Arquitectura de computadores en Ingeniería Informática (UGR)}

La asignatura de Arquitectura de Computadores se imparte en el segundo curso del grado en Ingeniería Informática de la Universidad de Granada. Se trata de una asignatura obligatoria cuyos objetivos y contenidos se han descrito en [1]. En [3] se proporciona un marco conceptual para la asignatura, y su guía docente se puede encontrar en [5]. La adaptación al EEES de los contenidos de arquitectura de computadores que se impartían en las titulaciones de Ingeniería informática previas supuso, aparte de una reducción considerable de créditos de docencia presencial de teoría y prácticas, la incorporación de ejercicios voluntarios de evaluación continua y la realización de pruebas para cada uno de los temas del programa. En un principio, el porcentaje de la nota final que correspondía a estas pruebas de evaluación era bastante pequeño: uno o dos puntos a lo sumo, y en la mayoría de los casos, eran puntos que se añadían a la nota final, solo si ésta alcanzaba un porcentaje mínimo de la nota máxima. En realidad, cuando se incluyeron estas pruebas de evaluación continua en las asignaturas de arquitectura de computadores, se pensaba más bien en que los estudiantes recibiesen realimentación acerca de su aprendizaje sobre los contenidos y las competencias que iban alcanzando en la asignatura. Se trataba de llevar a cabo la denominada evaluación formativa.

Sin embargo, una de las consecuencias de los estudios y discusiones sobre metodologías docentes que tuvieron lugar con motivo de la implantación del EEES fue la incorporación de la evaluación continua en prácticamente todas las asignaturas de todos los planes docentes. Se ha considerado que mediante la realización de actividades de evaluación continua se daba respuesta al problema que planteaban los exámenes finales únicos en cuanto a su limitación para evaluar todos los resultados de aprendizaje que se establecen en las guías. Si se pretende que los estudiantes sean capaces de resolver determinado tipo de problemas, es conveniente que se les planteen relaciones de problemas y se discutan en clase. En la mayoría de las asignaturas en carreras científicas o técnicas ya se acostumbraba a acompañar cada tema con una relación de problemas cuya resolución se aborda en clase. En grupos 
con un número moderado de estudiantes, la resolución de problemas en clase, las discusiones en seminarios, la aplicación esporádica de cuestionarios, o la propia formulación de preguntas en el desarrollo de las clases teóricas permite conocer a los estudiantes con cierto detalle e implementar la evaluación continua sin tener que realizar un número considerable de exámenes.

El problema es que, en grupos de clase numerosos (en el caso de los grupos de teoría ha habido años con grupos de hasta cien estudiantes), la única forma viable de implementar la evaluación continua implica que esté basada prácticamente solo en exámenes. Si además tenemos en cuenta que, en cada cuatrimestre, un estudiante debe cursar simultáneamente un número considerable de asignaturas, la competencia entre asignaturas por el interés y el trabajo del estudiante es un factor que debe tenerse en cuenta al planificar la asignatura. Esta situación nos hizo cambiar, hace un par de cursos, la forma en que se organizaba la evaluación de los contenidos de la asignatura. Por un lado, se incrementó el valor de las notas de evaluación continua: 4 puntos para la evaluación continua de teoría, que se obtenían tras cuatro pruebas de un punto cada una, realizadas al finalizar cada uno de los temas de teoría, y 2 puntos para la evaluación continua de las prácticas de la asignatura, que se obtenían a partir de las respuestas a cuestionarios que se pasaban en la clase práctica, más las calificaciones de las memorias de prácticas. Hay que tener en cuenta que los grupos de prácticas son menos numerosos que los de teoría: tienen unos 25 estudiantes.

Aparte de la evaluación continua, la reforma de Bolonia promueve que el profesor aplique la metodología docente más adecuada para los contenidos de la asignatura dando más relevancia al papel del estudiante como protagonista de su propio aprendizaje. En general, se favorece el aprendizaje basado en problemas y se intenta reducir el protagonismo de la lección magistral, que se suele identificar con el aprendizaje memorístico por parte del estudiante, y que se considera que puede ser sustituida por la disponibilidad de material en internet y a través las plataformas que suelen utilizarse para la docencia de las asignaturas. Sin embargo, el hecho de que se disponga del material de la asignatura a través de la correspondiente plataforma docente, no está contribuyendo de forma totalmente positiva al aprendizaje [7]. Así, aunque el aprovechamiento de las tecnologías de la información y las comunicaciones ha sido un elemento esencial para mejorar la calidad de la enseñanza universitaria, y plataformas docentes de fácil acceso y uso, como SWAD [6], han contribuido a difundir y acceder a material docente, mejorar la comunicación profesor-estudiante, y facilitar la gestión de los grupos, entre otras facetas de la docencia, dado que se dispone de las transparencias de clase y de las soluciones a problemas propuestos, muchos estudiantes no se preocupan de tomar apuntes ni de trabajar sobre otros problemas no resueltos de la asignatura. Simplemente, consideran que en su momento podrán asimilar la información, cuando vaya aproximándose el examen. Como si se tratase de copiar un fichero desde un directorio (en el disco) a otro (en el cerebro).

Sin embargo la neurociencia nos dice que nuestra memoria no funciona como el disco duro de nuestros computadores [8]. De hecho, los modelos más aceptados actualmente indican que el aprendizaje se construye, y la memorización es el primer paso de un proceso de síntesis personal de lo que se ha leído, escuchado, visto, etc., 
que implica creatividad y juicio. Los eventos que se introducen en la memoria a corto plazo se transforman en pensamientos memorizados y pasan a la memoria a largo plazo una vez transcurrido un cierto tiempo. Estos dos tipos de memoria se basan en procesos biológicos diferentes y se ha puesto de manifiesto que el paso a la memoria a largo plazo no sólo cambia la concentración de neurotransmisores para reforzar ciertas conexiones, sino que también aparecen nuevas sinapsis, implicando cambios tanto anatómicos como bioquímicos. Así, además de que debe activarse un gen para producir las proteínas correspondientes y responder al proceso de aprendizaje, la consolidación de la memoria a largo plazo implica una relativamente larga cadena de interacciones entre el hipocampo y el córtex cerebral, en cuya base está la atención.

Por otro lado, el acceso a la información que proporciona una página web conlleva una considerable carga cognitiva [7]. Cuando se accede a información en hipertexto aparecen opciones que pueden visitarse o no. El orden en el que se van visitando debe decidirse a medida que se va accediendo a la información según nos resulte más o menos interesante. El acceso a la información en este caso necesita un nivel de atención considerable, y nuestra memoria de trabajo estará expuesta a un número mayor de estímulos que si esa información nos llegara de forma lineal, como ocurre cuando accedemos a ella a través de un libro, o se nos proporciona estructurada de forma adecuada en una clase magistral.

Por tanto, seguimos pensando que las clases magistrales son importantes para el desarrollo de la docencia y recomendamos la toma de apuntes y el uso de libros de texto. Consideramos que la clase magistral tiene efectos bastante positivos si se introducen elementos que promuevan la participación del estudiante y la resolución de ejercicios y problemas con los que se va ilustrando la utilidad de los conceptos explicados. Los problemas de las clases magistrales surgen con la masificación de los grupos y la falta de relación entre explicaciones de teoría y clases de problemas que deberían estar completamente imbricadas.

\section{Docencia de Arquitectura de computadores en el confinamiento por la COVID-19}

La pandemia ha afectado a la asignatura en dos aspectos importantes, por una parte la impartición adecuada de las clases magistrales, y por otra la realización de pruebas de evaluación continua no presenciales. En cuanto a las clases magistrales, la existencia de plataformas de videoconferencia ha supuesto una ayuda considerable. Las clases se han impartido a través de la herramienta Meet de Google GSuite, que permite la grabación de las sesiones de clase, y su difusión a través de la herramienta Drive. La posibilidad de grabar las clases facilita al estudiante la asimilación y organización posterior de la información que se proporciona en clase. En principio, el estudiante recibiría la misma información del contenido de clase que le llegaría en una clase presencial y puede plantear dudas, intervenir en clase e interactuar con sus compañeros de clase a través de la herramienta de videoconferencia. De esta forma, una vez identificada la herramienta de videoconferencia a utilizar y tras 
familiarizarnos con su uso, se podría continuar explicando los contenidos de la teoría, como así ocurrió tras la primera semana de confinamiento. En esta rápida puesta en marcha de las clases no presenciales, la disponibilidad de plataformas docentes y herramientas (en nuestro caso Google GSuite UGR y SWAD [6]) ha sido importante, pero también han sido decisivas la colaboración entre los profesores de cada asignatura para transmitir experiencias de uso de este tipo de herramientas, y la formación recibida de entidades de la propia Universidad.

No obstante, las clases no presenciales plantean problemas importantes que deben tenerse en cuenta a la hora de mejorarlas. Por una parte están las posibles limitaciones de ancho de banda y de medios de que disponen los estudiantes, y las condiciones del entorno doméstico en el que se sigan las clases a distancia. Por otro lado, y quizá de más relevancia, se tienen los problemas relacionados con la comunicación limitada que existe entre estudiantes y profesor, y entre los propios estudiantes. Así, aunque es posible en principio tener imágenes de los estudiantes, en la práctica no se suelen utilizar imágenes de los participantes en las sesiones de videoconferencia para evitar posibles problemas derivados del ancho de banda que se requeriría en grupos de teoría con muchos estudiantes. Igual que sucede en las clases presenciales muy numerosas, la participación de los estudiantes en las clases no suele ser muy frecuente y debe interpelarse con demasiada frecuencia a los estudiantes para que pregunten. Algo que, ciertamente, resulta más complicado en una videoconferencia. La realimentación que el profesor recibe acerca del interés de sus estudiantes por lo que está explicando y sobre el grado de comprensión que está alcanzando su explicación, se ve reducida considerablemente con respecto a lo que sucede en las clases presenciales. Además, es bastante más complicado mantener la atención de los estudiantes, que siguen la clase a través de sus ordenadores, que les permiten navegar en Internet o simplemente ejecutar otras aplicaciones. También es difícil introducir cambios en el contenido de la clase, y llevar a cabo desarrollos sobre la marcha, ante los "silencios" de la clase cuando lo que se está explicando no se está entendiendo. Evidentemente, disponer de dispositivos con funciones de pizarra electrónica permite añadir nueva información a la que se recoge en las presentaciones que se tienen preparadas, pero determinar el momento en que debe hacerse requiere cierta preparación adicional a la que implica una clase presencial. Para concluir esta enumeración de las consecuencias de las clases no presenciales, no se pueden olvidar las limitaciones que existen para la comunicación entre los propios estudiantes, que suelen encontrar en esa interacción un apoyo esencial para resolver dudas que se les plantean al estudiar, y constituye la base de la importante dimensión social que tiene toda carrera universitaria. Consideramos que es esencial facilitar el contacto entre los compañeros de clase a través de las correspondientes herramientas de video-llamada y de trabajo cooperativo. Una forma de facilitar el intercambio estrecho de experiencias entre los integrantes de los grupos de clase es la configuración de grupos con pocos estudiantes, que "coincidan” frecuentemente en las clases no presenciales de sus asignaturas.

Incluso siendo muy relevantes los problemas que plantean las clases no presenciales, la parte más complicada en la adaptación de la docencia al escenario de confinamiento resultante de la COVID19 ha resultado ser la implementación de las 
pruebas de evaluación. Las plataformas docentes que hemos podido utilizar (en nuestro caso SWAD) incluyen herramientas que permiten gestionar bases de datos de preguntas y automatizar la realización y corrección de pruebas, y han resultado de gran ayuda. No obstante, la principal dificultad surge al tener que garantizar la autoría del examen: que el examen ha sido hecho por el estudiante correspondiente, en las condiciones en que debía hacerse respecto al uso de material de apoyo y, por supuesto, la ausencia de copias. Esto nos ha llevado a considerar la necesidad de tener en cuenta ciertas cuestiones éticas en la formación de nuestros estudiantes, tal y como se describe en la Sección 4.

\section{4 Ética y evaluación de aprendizajes durante la COVID-19}

Cuando las clases empezaron a impartirse de manera no presencial, una de las primeras inquietudes que afloraron entre los estudiantes fue la relativa a los exámenes, sobre todo una vez se constató que se podría continuar impartiendo la asignatura a través de videoconferencias, y que se podrían seguir los contenidos a través del material disponible en transparencias, libros de texto de teoría y de problemas resueltos $[9,10]$. Una parte considerable de la calificación de la asignatura proviene de las pruebas que se realizaban presencialmente tras cada tema, y en el momento en que se decretó el confinamiento solo se había realizado la correspondiente al primer tema. Los estudiantes planteaban frecuentemente si se continuarían realizando dichas pruebas para los restantes temas. Las pruebas se llevaban a cabo a través de la herramienta de test de la plataforma SWAD que, si bien permitía una elaboración eficiente de pruebas a partir de la selección aleatoria de preguntas contenidas en una base de datos que se podía ir ampliando, y facilitaba la corrección y el análisis de las pruebas, no incorporaba recursos muy sofisticados para su aplicación no presencial. Estaba pensada más como herramienta para generar pruebas de auto-evaluación y cuando se aplicaba para la evaluación continua se hacía como un examen presencial, con la supervisión del profesor. También hay que tener en cuenta que el establecimiento de las condiciones en las que se podría llevar a cabo una evaluación no presencial generó en su momento cierta controversia, y de hecho las resoluciones correspondientes del rectorado de nuestra Universidad llegaron bastante después de que se comenzasen a impartir las clases no presenciales, justo cuando ya era inminente el comienzo del periodo de exámenes. Así, por ejemplo, los estudiantes mostraban un amplio rechazo a las de herramientas de proctoring [11,12], de las que por otra parte no se disponía, e incluso al uso de cámaras que les estuviesen grabando durante el examen.

En cualquier caso, a pesar de las limitaciones para evitar copias que tenía la herramienta de exámenes de que se disponía, y aunque, como se ha dicho, ni se disponía de herramientas de proctoring, ni se podían utilizar, se decidió continuar la aplicación de pruebas de evaluación continua, sencillamente apelando a la responsabilidad y honradez de los estudiantes. Teniendo en cuenta las características de las herramientas que se utilizarían para elaborar y aplicar las pruebas no presenciales estaba clara la posibilidad de copiar en las correspondientes pruebas de evaluación continua. Sin embargo dada la situación especial (de crisis) en la que nos 
encontrábamos, se pensó que habría una respuesta clara y mayoritaria hacia la honradez ante el dilema moral que se planteaba: mantener un comportamiento altruista y no copiar pensando en el beneficio común de poder disponer de calificaciones de evaluación continua, o mostrar un comportamiento egoísta y copiar, a pesar de que se pudiese anular la evaluación continua. En cualquier caso, resultaría ilustrativo comprobar el comportamiento ético de nuestros estudiantes aunque estuviésemos en los momentos críticos de una pandemia.

Finalmente, la media de las calificaciones obtenidas por los estudiantes en las sucesivas pruebas de evaluación continua de los tres temas que faltaban creció considerablemente. Si bien hubo estudiantes con calificaciones que se mantuvieron dentro de una línea similar a la que correspondía con su nota en la primera prueba, manteniendo un comportamiento altruista como correspondía, también hubo un número significativo de estudiantes que alcanzaron calificaciones próximas a la máxima en cada una de las pruebas de evaluación continua no presenciales que faltaba realizar.

Por un lado, estas tendencias observadas en los resultados de las pruebas de evaluación continua, nos llevaron a implementar ciertos cambios en los exámenes de teoría y prácticas de todo el contenido de la asignatura (los denominados exámenes finales de la asignatura), dado que también tendrían que hacerse de forma no presencial. Se tuvo en cuenta para ello, el documento [13] del Ministerio de Universidades, donde incluso ya se indica que, "en el caso de los exámenes de tipo test o respuesta corta no existe una tecnología que pueda garantizar completamente la autoría y evite la realización de actos fraudulentos durante la realización de las pruebas”, pero existen recomendaciones para reducir su impacto. El examen se dividió en partes, presentando las preguntas de cada parte de forma sucesiva, sin posibilidad de retroceder a partes anteriores y ajustando el tiempo de respuesta. La detección de copias en el examen final hizo que, en el examen de la convocatoria extraordinaria, fuese obligatorio para el estudiante mantener la cámara activa durante todo el examen, aunque no se grababa nada.

Con frecuencia, los estudiantes han planteado su descontento con los procedimientos de examen que, según ellos, resultan de una excesiva preocupación de los profesores por evitar copias en los exámenes. Es lógica la preocupación por las copias, puesto que los profesores debemos garantizar que las calificaciones sean justas y reflejen el aprendizaje alcanzado por cada estudiante en las competencias correspondientes a la asignatura. Si no se hubieran producido copias, o los propios estudiantes las hubieran denunciado, los profesores no hubiéramos tenido que usar en los exámenes estrategias que, por otro lado, hacen más compleja su corrección. En cualquier caso, la solución al problema de las copias se debería buscar en la motivación de nuestros estudiantes, más que en una especie de “carrera armamentística” entre técnicas de copia y estrategias para evitarlas y detectarlas. Se podría aprovechar esta situación de crisis motivada por la pandemia, para que los estudiantes cooperen y contribuyan al bien común de la calidad docente, a pesar de que un comportamiento egoísta les pueda parecer que proporciona beneficios individuales más inmediatos. No obstante, algunos trabajos [14] sobre la generación de comportamientos cooperativos ante 
dilemas como el que plantean las copias en los exámenes, ponen de manifiesto que hay más probabilidad de cooperación voluntaria en los grupos pequeños que en los grandes, y no suele poderse mantener en grupos que superan un tamaño crítico.

Como consecuencia de todo esto, también hemos llegado a la conclusión de que, a través de la docencia que impartimos, conviene dar una mayor relevancia a la formación ética de nuestros estudiantes. Un ingeniero debería mostrar siempre un comportamiento ético intachable puesto que los resultados de su actividad profesional tendrán incidencia social. Los ingenieros que formamos deben ser objetivos y veraces, formarse para ser competentes en la toma de decisiones dentro de su profesión, y ser honrados en la propuesta de soluciones eficaces y seguras en una sociedad orientada hacia un desarrollo sostenible y justo.

\section{Conclusiones y propuestas para el futuro}

En este artículo se analizan algunos de los problemas que ha planteado la docencia no presencial de la asignatura Arquitectura de Computadores, de segundo del grado en Ingeniería Informática de la Universidad de Granada, y se describen algunas propuestas para darles respuesta.

Una de los aspectos que se ha manifestado como fundamental para mantener una calidad docente adecuada, incluso en situaciones tan difíciles como la vivida durante el confinamiento por la COVID19, es el número de estudiantes que constituyen los grupos, tanto de teoría como de prácticas. Grupos muy numerosos no solo dificultan la docencia en circunstancias normales (hacen que se deba estudiar la escalabilidad de cualquier estrategia docente que se quiera implementar) sino que afectan negativamente a la resiliencia y adaptabilidad ante imprevistos como el que hemos tenido, al tiempo que hacen menos probable que surjan comportamientos colaborativos entre los estudiantes, muy útiles ante problemas como, por ejemplo, los derivados de la necesidad de realizar exámenes no presenciales.

Si bien la reforma de Bolonia planteaba la formación continua, en grupos muy numerosos esa recomendación se ha implementado más como la aplicación de pruebas, frecuentemente de tipo test, y ejercicios a resolver por los estudiantes, que se ven sometidos a un número excesivo de exámenes correspondientes a las asignaturas que están cursando cada cuatrimestre. En grupos pequeños, el profesor puede llegar a conocer la evolución de los estudiantes a lo largo del cuatrimestre que suele durar una asignatura, y es viable la realización de seminarios a través de los que se pueden evaluar las competencias de los estudiantes sin tantos exámenes. Se pone así de manifiesto la relación entre la inversión económica realizada y la calidad de la formación.

En general, son esenciales el trabajo en equipo y colaboración, no solo entre estudiantes, y entre profesores, sino también entre estudiantes y profesores. Precisamente en estos momentos en los que el contacto directo es tan difícil, por no decir imposible y/o desaconsejable, es evidente la necesidad de herramientas de 
enseñanza no presencial adecuadas, en particular las que permitan realizar con garantías la evaluación no presencial.

\section{Agradecimientos}

Muchas gracias a nuestros compañeros Francisco Barranco, Maribel García Arenas, Juan José Escobar, Jesús González Peñalver, Niceto Luque, Christian Morillas, Gustavo Romero, y Jorge Sánchez por compartir su trabajo en la docencia de Arquitectura de Computadores en el segundo cuatrimestre del Curso 2019-2020. También agradecemos su apoyo a Miguel Damas, Director del Departamento de Arquitectura y Tecnología de Computadores, y a Antonio Cañas su herramienta SWAD. Se ha recibido financiación del proyecto PGC2018-098813-B-C31 (Ministerio de Ciencia, Innovación y Universidades y fondos FEDER).

\section{Referencias}

1. Ortega, J.; Anguita, M.:”Arquitectura de Computadores en seis créditos ECTS”. Enseñanza y Aprendizaje de Ingeniería de Computadores, No.2, pp.13-25, 2012.

2. Ortega, J.; Anguita, M.; González, Jesús; Damas, M.:”Reflexiones tras los primeros cursos de la asignatura Arquitectura de Computadores”. Enseñanza y Aprendizaje de Ingeniería de Computadores, No.6, pp.5-17, 2019.

3. Ortega, J.; Anguita, M.:”Relatos, Mapas Conceptuales y Arquitectura de Computadores”. Enseñanza y Aprendizaje de Ingeniería de Computadores, No.4, pp.89-102, 2014.

4. Ortega, J.; Anguita, M.; Prieto, A.; Cañas, A.; Damas, M.; Diaz, A.F.; Fernández, J.; González, J.: "La hoja de ruta de la ingeniería de computadores al final de la ley de Moore y el escalado de Dennard”. Enseñanza y Aprendizaje de Ingeniería de Computadores, No.9, pp.5-27, 2019.

5. Guía docente de Arquitectura de Computadores (grado en Ingeniería Informática de la UGR). https://atc.ugr.es/sites/departamentos_files/departamentos_atc/public/inline-

files/GuiasDocentes_20_21/gii_arquitectura_computadores_2020-2021.pdf.

6. SWAD (Sistema Web de Apoyo a la Docencia), swad.ugr.es, 2020.

7. Machado Trujillo, C.:”Aprendizaje con nuevas tecnologías: una mirada desde la neurociencia y la psicología cognitiva”. III Jornadas de la Innovación Educativas en el ámbito de las TIC, pp.235-242. Las Palmas de Gran Canaria, 17-18 de Noviembre, 2016.

8. Kandel, E.R.:"En busca de la memoria: una nueva ciencia de la mente". Katz Editores, 2007.

9. Ortega, J.; Anguita, M.; Prieto, A.:”Arquitectura de Computadores”. Ed. Thomson-Paraninfo, 2005.

10. Anguita, M.; Ortega, J.:"Fundamentos y Problemas de Arquitectura de Computadores". Editorial Técnica Avicam, 2016

11. Cramp, J.; Medlin, J.F.; Lake, P.; Sharp, C.:"Lessons learned from implementing remotely invigilated online exams, Journal of University Teaching \& Learning Practice, 16(1), 2019.

12. Alessio, H. M.; Malay, N.; Maurer, K.; Bailer, A. J.; Rubin, B.: "Examining the effect of proctoring on online test scores”, Online Learning 21 (1), 2017.

13. González, M.; Marco, E.; Medina, T.:’Informe de iniciativas y herramientas de evaluación online universitaria en el contexto del Covid-19”. Gabinete del Ministro. Ministerio de Universidades, 2000.

14. Kollock, P.: “Social Dilemmas: The Anatomy of Cooperation”. Annual Review of Sociology, Vol. 24, pp. 183-214. https://doi.org/10.1146/annurev.soc.24.1.183, 1998. 